\title{
Modulation of Protein Quality Control Systems as Novel Mechanisms Underlying Functionality of Food Phytochemicals
}

\author{
Kohta Ohnishi, Kazuhiro Irie, and Akira Murakami ${ }^{\dagger}$
}

Division of Food Science and Biotechnology, Graduate School of Agriculture, Kyoto University, Kyoto, 606-8502, Japan

${ }^{\dagger}$ Corresponding Author: Akira Murakami, PhD, Assistant Professor, Division of Food Science and Biotechnology, Graduate School of Agriculture, Kyoto University, Kyoto 606-8502, Japan

Submission date: May 8, 2013; Acceptance date: October 23, 2013; Publication date: October 30, 2013

\begin{abstract}
$\underline{\text { ABSTRACT }}$
Background: Phytochemicals are secondary metabolites of plants that are produced for their defense against environmental stresses, such as polyphenols, which are considered to play a major role in protection against ultraviolet (UV) light-induced oxidative damage, as well as anti-fungal and anti-microbial activities. In addition, there is a great body of evidence showing that phytochemicals exhibit a wide array of physiological activities in humans. Accumulated data show that the bioavailability of most, if not all, phytochemicals is quite poor and their substantial biotransformation after ingestion has also been noted. Thus, they are characterized as non-nutritive xenobiotics in animals, and the question of why phytochemicals, which are produced for plant self-defense, have beneficial effects in humans is quite intriguing. Meanwhile, stress-induced denaturing of cellular proteins greatly affects their tertiary structure and critically disrupts their biological functions, occasionally leading to aggregation for the onset of some pathology. Many recent studies have indicated that protein quality control (PQC) systems play key roles in counteracting 'proteo-stress', which is comprised of several processes, including protein refolding by heat shock proteins (HSPs) and degradation of abnormal proteins by the ubiquitin-proteasome system as well as autophagy.
\end{abstract}


Objective: Phytochemicals are xenobiotics, thus their biochemical interactions with animal proteins are considered to occur in a non-specific manner, which raises the possibility that some phytochemicals cause proteo-stress for activating PQC systems. Because their status is thought to be a critical determinant of homeostasis, the physiological functions of phytochemicals may be partially mediated through those unique systems. The present study was thus undertaken to address this possibility.

Methods and Results: We focused on zerumbone (ZER), an electrophilic sesquiterpene present in Zingiber zerumbet Smith (shampoo ginger). This agent has been reported to exhibit various bioactivities, including anti-inflammation and cancer prevention[1,2]. Treatment of Hepa1c1c7 mouse hepatoma cells with ZER resulted in marked up-regulation of multiple HSPs, such as HSP40 and HSP70. Furthermore, oral administration to the nematode Caenorhabditis elegans and SD rats increased the expressions of some HSPs[3]. Interestingly, ZER also increased proteasome activity in Hepa1c1c7 cells, which was accompanied with up-regulation of $\beta 5$, a major proteasome functional protein. In addition, the agent notably up-regulated the expressions of several pro-autophagic markers, including p62 and microtubule-associated protein 1 light-chain 3 (LC3)-II[4]. Experiments with biotin-labeled ZER as well as a specific antibody against ZER-adduct proteins revealed that it binds numerous cellular proteins in a non-specific manner. Along a similar line, incubation with ZER led to formations of p62-conjugated proteins and aggresomes. Together, these results suggest that ZER causes proteo-stress for potentiating the integrity of PQC systems. In support of this notion, ZER-bound proteins have been suggested to be partially recognized by HSP90, leading to dissociation of heat shok factor 1 (HSF1) from HSP90 for inducing multiple HSP genes. Next we speculated that mild chemical stress by ZER may exert beneficial effects, since ZER-bound proteins were time-dependently degraded, suggesting that defense capacity was amplified to a great level as compared with the non-treated condition. As expected, ZER conferred thermoresistance to Caenorhabditis elegans (C. elegans) and suppressed the proteo-toxicity of 4-hydroxy-2-nonenal, a potent electrophile produced through a lipid peroxidation process, in a p62-dependent manner. We then screened a number of nutrients and phytochemicals for their HSP70 inducibility, and found that certain phytochemicals, such as curcumin, phenethyl isothiocyanate, ursolic acid, and lycopene, were significantly active, whereas most nutrients were virtually inactive. These results may be associated with the fact that phytochemicals, but not nutrients, are foreign chemicals to animals, as noted above. 
Conclusion: Up-regulation of antioxidant and xenobiotics-metabolizing enzymes has been reported to be an adaptive response in animals exposed to phytochemicals. Our present results imply that the process also increases the capacity to counteract proteo-stresses through activation of PQC systems. This putative phenomenon, representing the concept of hormesis[5], may be associated with mechanisms underlying the physiological functions of phytochemicals. Therefore, chronic ingestion of this class of chemicals may result in 'chemical training', in which self-defense systems are continuously activated for adaptation to phytochemical-driven stresses.

Key words: heat shock proteins, ubiquitin-proteasome system, autophagy, C. elegans

\section{BACKGROUND:}

Natural compounds derived from plants are divided into 'primary metabolites' and 'secondary metabolites'. Primary metabolism is common pathway of biosynthesis among any species, which provides essential molecules for their survivals such as polysaccharides, lipids, proteins, and nucleotides and so on. They are known to play the major roles on the basic biological activities such as energy storage, catalyst of biological reaction, and transduction of genetic information. On the other hand, secondary metabolism is a specific process to a particular species, which provides various natural compounds for adaptation in response to the environmental stresses[6]. For example, some plants biosynthesize polyphenols with high UV absorbing activities in their leaf epidermis and pericarp, which are considered to play a key role in protection against UV light-induced oxidative damage. Likewise, plants are known to produce a variety of compounds with anti-fungal, microbial, and insect activities in response to erosion stresses[7].

Recently, plant secondary metabolites, some of which are contained in food materials, have been revealed to possess a wide range of physiological activities in mammals. Many epidemiologic studies indicated that daily intake of food phytochemicals reduces the risk of onsets of diverse diseases including cancer and neurodegenerative disorders in human[8,9]. Correspondingly, there has been accumulated evidence showing that plant secondary metabolites exhibit various bioactivities relating to anti-oxidation, anti-inflammation, and detoxification, in mammalian cell lines as well as rodents[10,11]. Recent mechanistic studies have revealed that some phytochemicals are bound to multiple signaling molecules in mammals for the modulation of signal transduction pathways which are associated with development of various diseases[12]. However, the biological reason why plant secondary metabolites exhibit such bioactivities in 
mammals remains to be fully explained. Considering that phytochemicals are originally produced by plants for their survivals, they should be recognized to be xenobiotic compounds in mammals (Fig. 1). Indeed, bioavailabilities of phytochemicals in mammals have been revealed to be substantially poor, because they are subject to biological inactivation through detoxification mechanisms. For example, anti-carcinogenic sulforaphane (SFN), an isothiocyanate broadly distributed among cruciferous vegetables, is immediately metabolized to its glutathione conjugate by phase II detoxification enzymes and then excreted in urine[13]. This metabolic process is in contrast to that of glucose, which is actively absorbed via its specific transporter. However, it is interesting that molecular mechanisms underlying bioactivities of phytochemicals are partly associated with such xenobiotic properties.

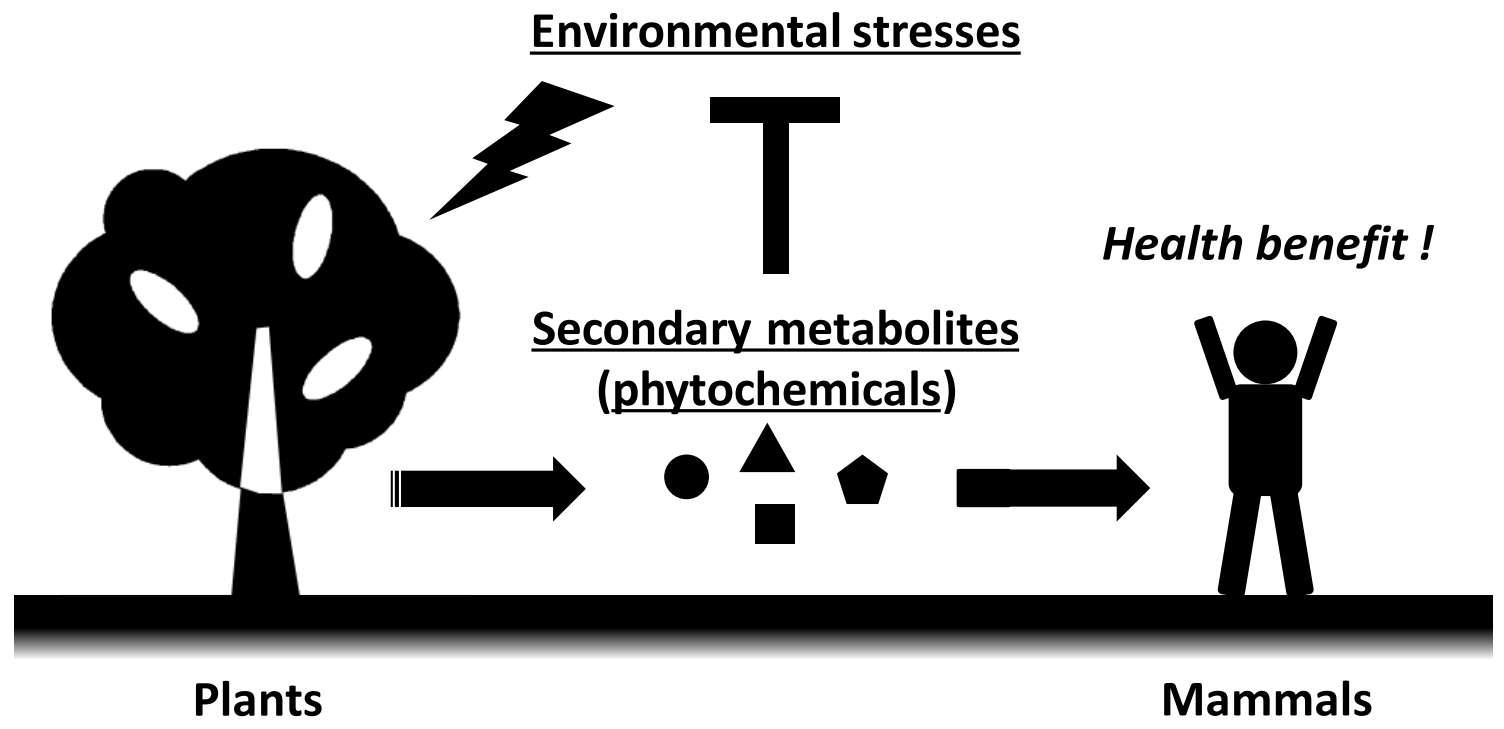

Figure 1. Why do phytochemicals exhibit bioactivities in mammals?

Plants produce secondary metabolites for their survivals in response to environmental stresses. Fundamental reasons why phytochemicals exhibit physiological functions in mammals remains to be fully answered.

Some electrophilic phytochemicals are known to activate nuclear factor-E2-related factor 2 (Nrf2) for inducing phase II detoxify enzymes, which lead to anti-oxidation and anti-carcinogenic properties[14,15]. Under a homeostatic condition, Nrf2, a key transcription factor for regulating the expressions of detoxifying and anti-oxidative enzymes, is inactivated by binding to Kelch-like ECH-associated protein 1 (Keap1). Only when cells are exposed to 
oxidative stress or electrophilic compounds, Nrf2 is allowed to translocate to nucleus and induces the target genes through modifications of reactive thiol groups of Keap1[16]. Thus, cells possess the mechanisms to reinforce their anti-oxidative and detoxifying abilities against certain biological stresses, although severe stresses can lead to their collapse. Such beneficial effects provided by mild biological stresses through activation of adaptation mechanisms are termed 'hormesis'[5]. Hormetic effects can be provided by not only oxidative stress. For example, radiation, a well-known carcinogenic stress, has been also reported to exhibit hormetic effects. Surprisingly, an epidemiological study has revealed that an average radiation dose of $0.4 \mathrm{~Sv}$ during a 9-20 year period dramatically reduced incidence of spontaneous cancer death by 97\%[17], implying a marked potential for health benefit.

Proteo-stresses such as heat shock denature biological proteins, which leads to alteration of their functions as well as tertiary structures. Under a severe denaturing condition, intracellular proteins are occasionally aggregated, which has been reported to be associated with aging and onsets of several disorders such as neurodegenerative disease and cancer[18]. Many recent studies have indicated that PQC systems play key roles in countering such proteo-stress, which is comprised multiple processes, including protein refolding by molecular chaperones and proteolysis by the ubiquitin-proteasome systems as well as autophagy (Fig. 2). Importantly, these PQC machineries are known to be activated by moderate proteo-stresses[19].

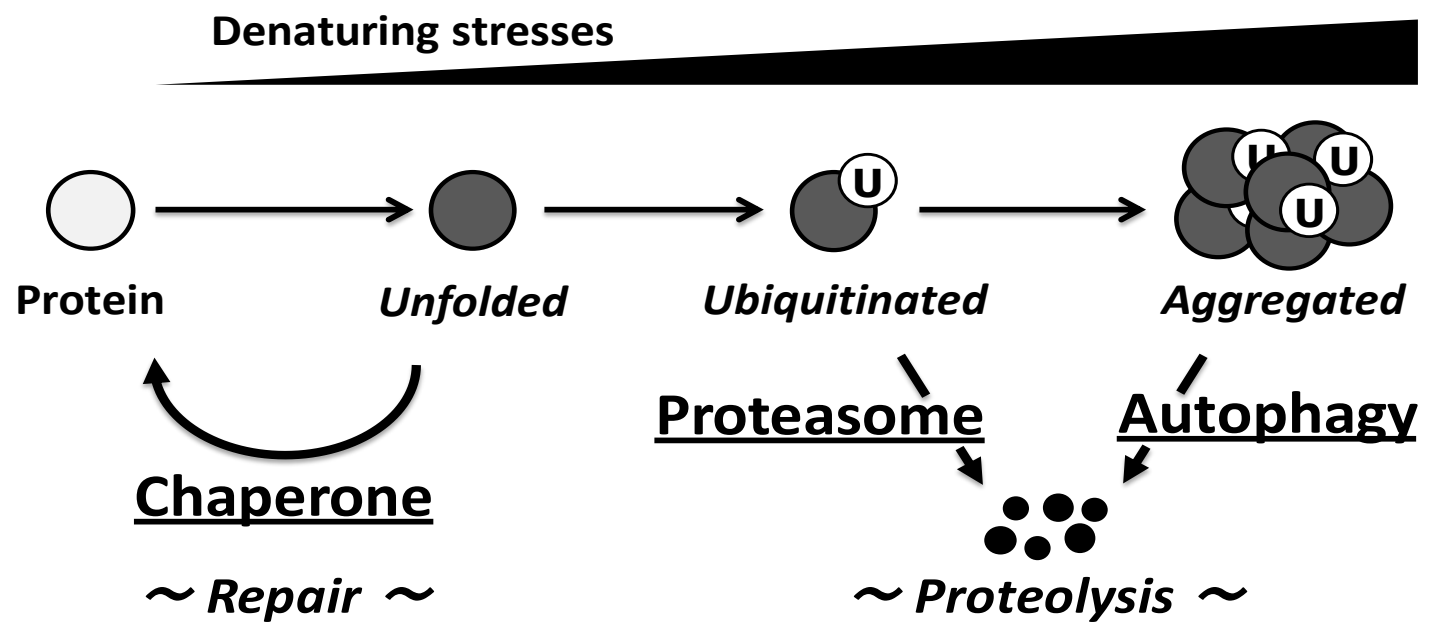

Figure 2. Protein quality control systems

Figure 2 Intracellular proteins are unfolded by denaturing stresses, followed by their ubiqutination and aggregation. Multiple protein quality control systems, including molecular 
chaperones, ubiquitin-proteasome systems, and autophagy, play key roles in countering such proteo-stress.

HSPs are major molecular chaperones highly conserved among all species from bacteria to humans. HSPs not only refold denatured proteins but also prevent their aggregation basically through binding to hydrophobic surfaces of denatured proteins. Importantly, HSPs expressions are up-regulated via heat shock response (HSR) as mentioned below. HSP90, a major constitutive HSP, is a negative regulator of the transcription factor HSF1, which regulates the expressions of inducible isoforms of HSPs such as HSP70 and HSP40. Upon exposure to stress, denatured proteins induce non-bound HSP90 recruitment, which enables HSF1 to transactivate the target genes[20]. Thus, proteo-stress at appropriate levels may reinforce PQC systems via HSR induction.

Excessively denatured proteins are occasionally ubiquitinated by carboxy terminus of Hsc70 interacting protein (CHIP), a chaperone-dependent E3 ligase, for their degradation[21]. The 26S proteasome, a multi-catalytic protease complex, is responsible for degradation of monomeric proteins. Ubiquitinated proteins are recognized by the $19 \mathrm{~S}$ regulatory subunit including Rpt1-6, and proteolysis occurs in the 20S catalytic core subunit consisting of $\alpha 1-7$ and ß1-7, which exhibits chymotrypsin, trypsin, and caspase-like activities[22]. Nrf2 has been reported to induce proteasome activity in response to oxidative and electrophilic stress. Microarray analysis showed that Nrf2 is responsible for induction of both 19S (Rpt2, Rpt5, Rpt11, etc.) and $20 \mathrm{~S}(\alpha 3, \alpha 6, \beta 3, \beta 5, \beta 7$, etc.) subunits[23]. On the other hand, autophagy is a bulk degradation system by which cytoplasmic materials are engulfed by double-membrane vesicles, known as autophagosomes, and delivered to lysosomes for degradation. It is known as an essential step in autophagy induction that conjugation of phosphatidylethanolamine to LC3 in generation of LC3-II associated with autophagosomes[24]. Recently, there is growing evidence that aggrephagy, the selective autophagic process for protein aggregates, is a highly selective PQC mechanism[18]. In aggrepahgy, p62 binds to both LC3 and ubiquitinated proteins to execute proteolysis, which is one of target genes of Nrf2.

\section{OBJECTIVE:}

Recent studies have revealed that phytochemicals specifically bind to proteins in mammalian cell lines. In 2004, Tachibana et al. identified a 67-kDa laminin receptor as a receptor for (-)-epigallocatechin-3-gallate, a major green tea polyphenol with chemopreventive activity[25]. Following this pioneering work, several studies of the target molecules of phytochemicals such 
as polyphenols and isothiocyanates have been presented. However, there are scant experimental data or insights regarding their binding selectivities. Considering that most, if not all, of these chemical structures are simple and small, it is reasonable to assume that they largely interact with proteins in a non-specific manner. In support of this notion, several isothiocyanates including SFN were shown to bind to unidentified multiple proteins in cultured cells[26,27], though the authors did not highlight those findings in their studies. Such non-selective bindings to proteins by phytochemicals are presumed to be potentially proteo-toxic, leading to activation of PQC systems under the appropriate conditions. This study was first undertaken to address this possibility of phytochemicals for exhibiting proteo-hormetic effects.

\section{METHODS AND RESULTS:}

Zerumbone (ZER) is a sesquiterpene derived from Zingiber zerumbet Smith (Zingiberaceae, shampoo ginger). In Southeast Asia, the rhizomes of this plant contain large amounts of ZER and have been used as a traditional medicine for analgesic and anti-inflammatory purposes. The bioactivities of ZER have been studied over the past two decades, which revealed its cancer preventive[2], anti-inflammatory[28], and detoxifying[15] activities, although the mechanisms underlying these activities are not fully clarified. Interestingly, $\square$-humulene, an analog of ZER lacking an $\alpha, \beta$-unsaturated carbonyl group, had less bioactivities in several studies[29], implying that this electrophilic moiety of ZER would be essential to exhibit its potent bioactivities. These studies raised the possibility of ZER to bind to nucleophilic groups in functional proteins covalently via Michael reaction (Fig. 3).

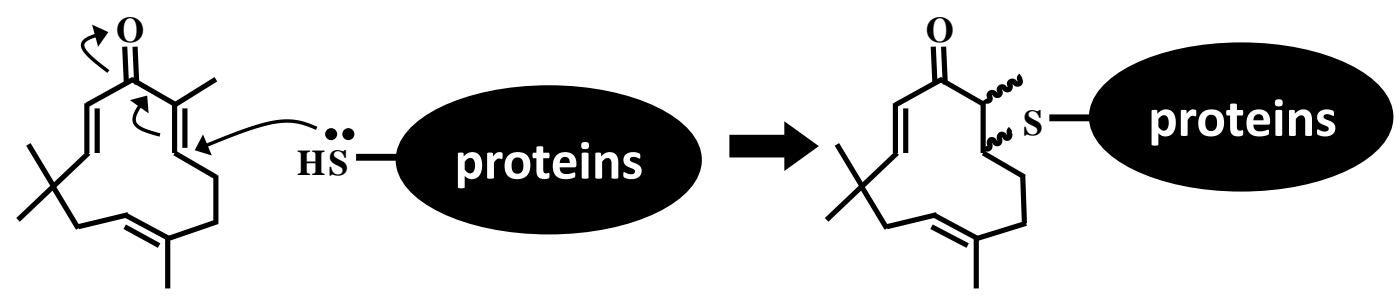

ZER

Figure 3. ZER is covalently bound to thiol groups in proteins.

At first, ZER-immobilized sepharose (SepZER) were prepared for the identification of cellular proteins bound to ZER. Cell lysates from RAW264.7 mouse macrophages were subjected to pull-down assay with SepZER, which revealed the covalent bindings of SepZER to 
various proteins. Western blot analysis revealed that SepZER was bound to possible key proteins for bioactivities of ZER such as Keap1 and homologous to human R-antigen (HuR), which regulates RNA stabilities of inflammatory genes[30]. Furthermore, incubation with cell lysates and $N$-ethylmaleinide, a specific thiol modifier, markedly suppressed the bindings of SepZER to these proteins, indicated that SepZER modified thiol groups of cellular key proteins including Keap1 and HuR[31].

Next, biotinylated ZER (BioZER) was chemically synthesized for the detection of its binding proteins in living cells. RAW264.7 cells incubated with BioZER were lysed for western blot analysis with HRP-conjugated avidin, which resulted in dramatic formation of BioZER-bound proteins. Then, cell lysates subjected to pull-down assay with avidin-immobilized sepharose were analyzed by western blot for identification of BioZER-bound proteins. Among 28 possible key proteins, which may be associated with bioactivities of ZER, examined in this experiment, almost all proteins (23 proteins), including Keap1 and HuR, were revealed to be bound to BioZER. Binding selectivities of BioZER to each protein were evaluated with band intensities in western blot analysis, which revealed that Keap1 possesses the highest binding potential to BioZER. As mentioned above, Keap1 is a negative regulator of Nrf2, an essential transcription factor for the expressions of anti-oxidative and detoxifying enzymes. Recent study also showed that heme oxygenase-1 induction by ZER was dependent on Nrf2[32]. Considering these findings, Keap1 might be one of the target proteins of ZER for its anti-oxidant and detoxifying bioactivities. Given that ZER was reported to modify and decrease cellular reduced glutathione[15], leading to oxidative stress, induction of anti-oxidative enzymes through modification of Keap1 can be considered to be one of hormetic effects induced by ZER.

In addition to identification of Keap1 as a target protein of ZER, numerous proteins were revealed to be modified by BioZER with less-selectivity. To confirm whether ZER itself also modifies multiple cellular proteins in a similar manner, an antibody against ZER-thiol adducts (anti-ZER Ab) were prepared. Hepa1c1c7 mouse hepatocytes treated with ZER were lysed for western blot analysis with anti-ZER Ab. Consistent with the results with BioZER, ZER modified numerous cellular proteins in a non-specific manner. Additionally, cells treated with ZER were immunostained with anti-ZER Ab, showing global distribution of ZER-thiol adducts throughout the cytoplasm and even in the nucleus. These data indicate substantially poor selectivity of ZER in modifying cellular proteins.

Non-specific protein modifications by ZER are presumed to be potentially proteo-toxic. Excessively denatured proteins are known to be ubiquitinated, followed by their aggregation, as 
mentioned above. Then, the possibilities of ZER to promote ubiquitination and aggregation of cellular proteins were examined. Hepa1c1c7 cells exposed to ZER were lysed for western blot analysis, showing its concentration-dependent ubiquitination of cellular proteins. Importantly, this phenomenon was scarcely seen in CHIP, a chaperone dependent E3 ligase, silencing cells, implying ZER denatures cellular proteins, followed by CHIP-dependent ubiquitination. To visualize protein aggregates, cells were stained with molecular rotor dye, resulting in the increase of cellular aggresome by ZER treatment. These results showed potential proteo-toxicity of ZER, which might be caused by non-specific protein modifications[4].

Given that ZER possesses proteo-toxic potential, it might induce proteo-hormetic effects for activation of PQC systems under the appropriate condition. At first, the possibility of ZER to induce HSR was examined in Hepa1c1c7 cells. As mentioned above, HSP90, a negative regulator of a transcription factor HSF1, was bound to denatured proteins for HSR induction. To detect the interaction between HSP90 and ZER-modified proteins, cell lysates treated with ZER were immunoprecipitated with anti-HSP90 antibody. Interestingly, multiple proteins modified by ZER were co-immunoprecipitated with HSP90, implying HSP90 might recognize ZER-modified proteins as denatured ones. To verify HSR after HSP90 recognition of ZER-modified proteins, HSF1 phosphorylation at Ser326, one of the residues crucial for its full activation[33], were semi-quantified by ELISA, which revealed that ZER significantly induced HSF1 phosphorylation. In accord with this observation, treatment with ZER resulted in increase of both mRNA and protein expressions of inducible form of HSPs such as HSP70 and HSP40 as examined by $\mathrm{RT}^{2}$-PCR and western blot analysis. Furthermore, HSP70-inducing activity of ZER was not seen in HSF1-silencing cells. These data indicates that ZER induced HSR through non-specific protein modifications in Hepa1c1c7 cells. Additionally, exposure of the nematodes (C.elegans) to ZER markedly increased the mRNA expression of HSP16.41, which is highly expressed in the intestines and pharynx. Also in livers of SD rats, oral administration of ZER significantly induced the protein expression of HSP70. These in vivo experiments demonstrated that this compound is orally active for inducing HSPs expressions[3].

Proteolysis machineries might be also activated for adapting to proteo-stress induced by ZER. The effect of ZER to proteasome activity was examined in Hepalc1c7, resulting in increase of cellular chymotrypsin-like activity. Western blot analysis showed that expression of $\square 5$, the 20S proteasome subunit essential for chymotrypsin-like activity, was up-regulated by ZER. On the other hand, autophagy activation was evaluated by western blot analysis of LC3-II, whose increase is a hallmark of this phenomenon, resulting in up-regulation of LC3-II by ZER. 
To confirm this autophagic response to ZER, cellular acidic vesicular organelles including autolysosomes and lysosomes were stained by acridine orange, which resulted in an increase of acidic vesicles by ZER. Additionally, ZER significantly induced some pro-autophagic genes including p62, an essential protein for aggrephagy. These results indicated that ZER also activated proteolysis machineries such as UPS and autophagy[4]. To verify whether ZER-modified proteins are degraded through these proteolysis mechanisms, cells were washed by PBS after ZER treatment, followed by incubation in ZER-free medium. Interestingly, ZER-modified proteins decreased in a time dependent manner, which was canceled by treatment with lactacystin, a proteasome inhibitor, or E64d and pepstatin A, autophagy inhibitors.

ZER was revealed to possess the ability of activating PQC systems (Fig. 4), suggesting that defense capacity against proteo-stress was amplified to a higher level as compared with the non-treated condition. Importantly, pretreatment of Hepa1c1c7 cells with ZER suppressed cellular protein modifications by 4-hydroxy-2-nonenal (HNE), a major lipid peroxidation product that is known to intensively modify cellular proteins[34]. In accord with this phenomenon, pretreatment with ZER also conferred a phenotype resistant to cytotoxicity by HNE, which was abolished in p62-silencing cells. These data implied that ZER reinforced the cellular defense capacity against proteo-stress through p62-dependent aggrephagy activation[4]. Also in vivo test with C.elegans, ZER administration significantly increased survival rates of nematodes after heat shock treatment[3].

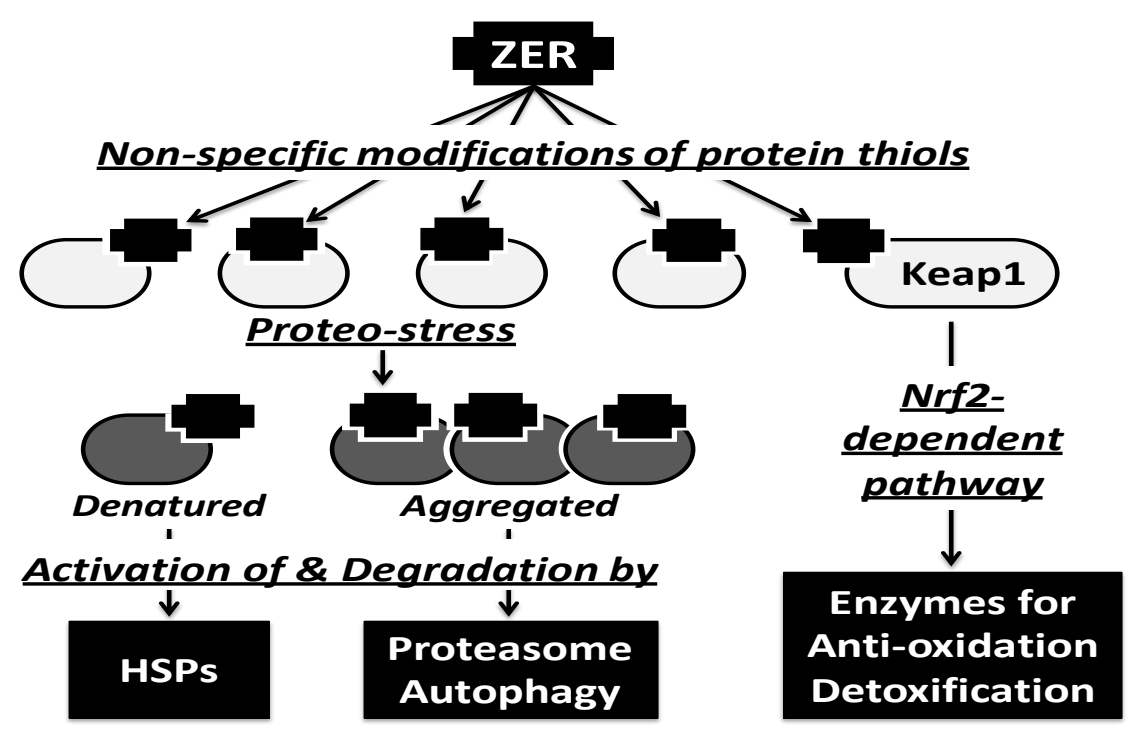

Figure 4. Non-specific protein modifications by ZER activated multiple self-defense systems including protein quality control systems via mild proteo-stress. 
Finally, HSP70 inducing activities of various natural compounds including plant primary and secondary metabolites were evaluated to examine whether this activity is limited to that seen with ZER. Among the tested nutrients, almost all compounds except for all-trans retinol and zinc chloride were inactive, implying that most nutrients are not recognized as xenobiotic stressors, because they are essential for mammalian homeostasis. Next, HSP70 inducibilities of phytochemicals including polyphenols and terpenoids were examined. Interestingly, most polyphenols examined in this experiment did not up-regulate HSP70, but rather tended to suppress it, whereas hydrophobic or electrophilic compounds such as ursolic acid, lycopene, isothiocyanate, and curcumin were also identified as potent HSP70 inducers[3]. These results support the hypothesis that non-specific protein modifications induce HSR, because both hydrophobicity and electrophilicity are major determinants for the non-selective interactions of chemicals with proteins.

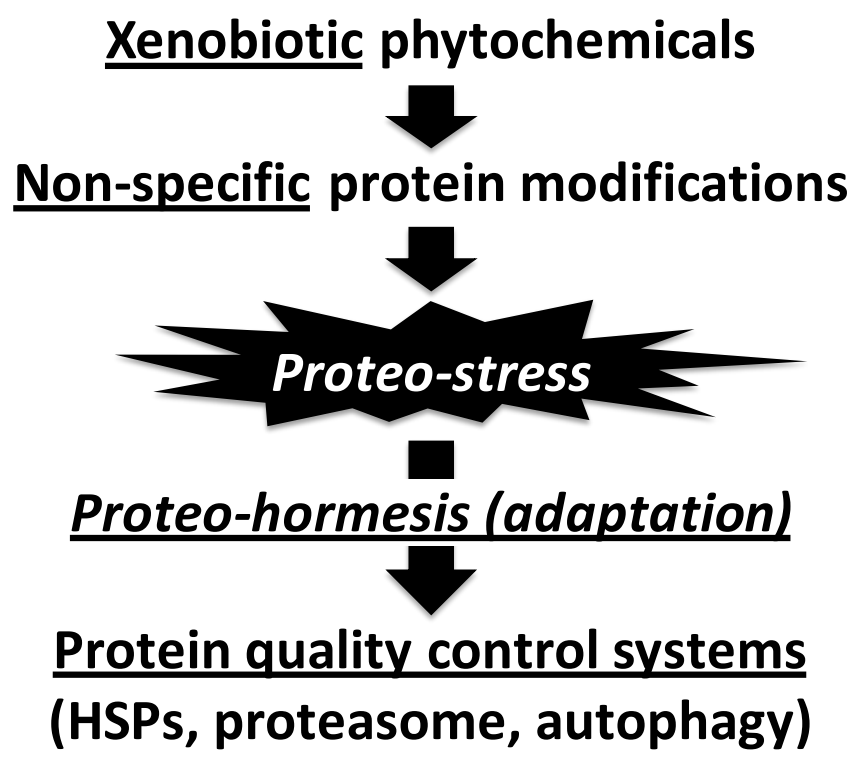

Figure 5. Proteo-hormesis induced by phytochemicals

\section{CONCLUSION:}

ZER, a chemopreventive sesquiterpene, was revealed to modify thiol groups in cellular proteins via its active moiety, $\alpha, \beta$-unsaturated carbonyl group[31]. Keap1, a negative regulator of $\mathrm{Nrf} 2$, was shown to be modified by ZER with relatively higher selectivity. Given that ZER has been shown to activate Nrf2 that is a key transcription factor for regulating the expressions of anti-oxidant and detoxifying enzymes, Keap1 is considered as one of target molecules of ZER, 
which might be one of hormetic mechanisms against oxidative stress caused by ZER. In addition to such selective modification, it was also clarified that numerous cellular proteins were modified by ZER with poor selectivity. Importantly, such non-specific modifications induce proteo-stress, leading to ubiquitination and aggregation of cellular proteins[4], which has been revealed to rather activate multiple PQC machineries, including HSPs[3], UPS, and autophagy[4]. This is the first study demonstrating proteo-hormetic effects induced by a phytochemical (Fig. 5). Hydrophobic or electrophilic phytochemicals other than ZER also possessed HSP70 inducing activities, but nutrients did not, suggesting the significance of their xenobiotic properties for activating PQCs. However, it is noted that there are some reports suggesting that HSR induction by phytochemicals is induced by modification of specific thiol groups in HSP90[27]. The substantial contribution ratio of non-specific protein modifications in HSR by phytochemicals remains to be answered.

Although it is needless to say that biological stresses can lead to collapse of homeostasis under the severe condition, an appropriate degree of stress might induce hormetic effects for activation of defense systems[5]. Recent reports have shown that dysfunction of PQC systems are associated with aging and various disorders[18,35]. A large number of food phytochemicals have been reported to exhibit a wide range of physiological functions, whose mechanisms might be basically associated with such proteo-hormetic effects. Therefore, chronic ingestion of this class of chemicals may result in 'chemical training', in which self-defense systems are continuously activated for adaptation to phytochemical-induced stresses.

\section{Competing Interests:}

The authors have no financial interests or conflicts of interest.

\section{Authors' Contributions:}

All authors contributed to this study.

\section{Abbreviations:}

BioZER, biotinylated ZER; C.elegans, Caenorhabditis. elegans; CHIP, carboxy terminus of Hsc70 interacting protein; HNE, 4-hydroxy-2-nonenal; HSF1, heat shock factor 1; HSP, heat shock protein; HSR, heat shock response; HuR, homologous to human R-antigen; Keap1, Kelch-like ECH-associated protein 1; LC3, microtubule-associated protein 1 light-chain 3; Nrf2, nuclear factor-E2-related factor 2; PQC, protein quality control; SepZER, ZER-immobilized 
sepharose ; SFN, sulforaphane; UV, ultraviolet; ZER, zerumbone

\section{Acknowledgements and Funding:}

We are grateful for our collaborator, as well as the previous and current graduate students in our lab. Our studies described in this article were partly supported by a Grant-in-aid for Scientific Research (C) (No. 23580164 to A.M.) and a Grant-in-aid for the Promotion of Science for Young Scientists (No. 22.3355 to K.O.) from the Ministry of Education, Culture, Sports, Science, and Technology of the Japanese Government.a Grant-in Aid for Scientific Rersearch (C) (A.M.) and a grant from Japan Society for the Promotion of Science Fellows (K.O.).

\section{REFERENCES:}

1. Murakami A, Takahashi D, Kinoshita T, Koshimizu K, Kim HW, et al. (2002) Zerumbone, a Southeast Asian ginger sesquiterpene, markedly suppresses free radical generation, proinflammatory protein production, and cancer cell proliferation accompanied by apoptosis: the alpha,beta-unsaturated carbonyl group is a prerequisite. Carcinogenesis 23: 795-802.

2. Kim M, Miyamoto S, Yasui Y, Oyama T, Murakami A, et al. (2009) Zerumbone, a tropical ginger sesquiterpene, inhibits colon and lung carcinogenesis in mice. Int $\mathbf{J}$ Cancer 124: 264-271.

3. Ohnishi K, Ohkura S, Nakahata E, Ishisaka A, Kawai Y, et al. (2013) Non-specific protein modifications by a phytochemical induce heat shock response for self-defense. PLoS One 8: e58641.

4. Ohnishi K, Nakahata E, Irie K, Murakami A (2013) Zerumbone, an electrophilic sesquiterpene, induces cellular proteo-stress leading to activation of ubiquitin-proteasome system and autophagy. Biochem Biophys Res Commun 430: 616-622.

5. Calabrese EJ, Iavicoli I, Calabrese V (2012) Hormesis: why it is important to biogerontologists. Biogerontology 13: 215-235.

6. Wink M (2003) Evolution of secondary metabolites from an ecological and molecular phylogenetic perspective. Phytochemistry 64: 3-19.

7. Treutter D (2005) Significance of flavonoids in plant resistance and enhancement of their biosynthesis. Plant Biol (Stuttg) 7: 581-591.

8. Hutchins-Wolfbrandt A, Mistry AM (2011) Dietary turmeric potentially reduces the risk 
of cancer. Asian Pac J Cancer Prev 12: 3169-3173.

9. Mandel SA, Amit T, Weinreb O, Youdim MB (2011) Understanding the broad-spectrum neuroprotective action profile of green tea polyphenols in aging and neurodegenerative diseases. J Alzheimers Dis 25: 187-208.

10. Nishiumi S, Miyamoto S, Kawabata K, Ohnishi K, Mukai R, et al. (2011) Dietary flavonoids as cancer-preventive and therapeutic biofactors. Front Biosci (Schol Ed) 3: $1332-1362$.

11. Zhang Y (2012) The molecular basis that unifies the metabolism, cellular uptake and chemopreventive activities of dietary isothiocyanates. Carcinogenesis 33: 2-9.

12. Hou DX, Kumamoto T (2010) Flavonoids as protein kinase inhibitors for cancer chemoprevention: direct binding and molecular modeling. Antioxid Redox Signal 13: 691-719.

13. Egner PA, Kensler TW, Chen JG, Gange SJ, Groopman JD, et al. (2008) Quantification of sulforaphane mercapturic acid pathway conjugates in human urine by high-performance liquid chromatography and isotope-dilution tandem mass spectrometry. Chem Res Toxicol 21: 1991-1996.

14. Zhang Y, Talalay P, Cho CG, Posner GH (1992) A major inducer of anticarcinogenic protective enzymes from broccoli: isolation and elucidation of structure. Proc Natl Acad Sci U S A 89: 2399-2403.

15. Nakamura Y, Yoshida C, Murakami A, Ohigashi H, Osawa T, et al. (2004) Zerumbone, a tropical ginger sesquiterpene, activates phase II drug metabolizing enzymes. FEBS Lett 572: $245-250$.

16. Kobayashi M, Yamamoto M (2005) Molecular mechanisms activating the Nrf2-Keap1 pathway of antioxidant gene regulation. Antioxid Redox Signal 7: 385-394.

17. Chen WL, Luan YC, Shieh MC, Chen ST, Kung HT, et al. (2007) Effects of cobalt-60 exposure on health of Taiwan residents suggest new approach needed in radiation protection. Dose Response 5: 63-75.

18. Lamark T, Johansen T (2012) Aggrephagy: selective disposal of protein aggregates by macroautophagy. Int J Cell Biol 2012: 736905.

19. Sorger PK (1991) Heat shock factor and the heat shock response. Cell 65: 363-366.

20. Baler R, Dahl G, Voellmy R (1993) Activation of human heat shock genes is accompanied by oligomerization, modification, and rapid translocation of heat shock transcription factor HSF1. Mol Cell Biol 13: 2486-2496. 
21. Murata S, Minami Y, Minami M, Chiba T, Tanaka K (2001) CHIP is a chaperone-dependent E3 ligase that ubiquitylates unfolded protein. EMBO Rep 2: 1133-1138.

22. Unno M, Mizushima T, Morimoto Y, Tomisugi Y, Tanaka K, et al. (2002) The structure of the mammalian 20S proteasome at 2.75 A resolution. Structure 10: 609-618.

23. Kwak MK, Wakabayashi N, Itoh K, Motohashi H, Yamamoto M, et al. (2003) Modulation of gene expression by cancer chemopreventive dithiolethiones through the Keap1-Nrf2 pathway. Identification of novel gene clusters for cell survival. J Biol Chem 278: 8135-8145.

24. Longatti A, Tooze SA (2009) Vesicular trafficking and autophagosome formation. Cell Death Differ 16: 956-965.

25. Tachibana H, Koga K, Fujimura Y, Yamada K (2004) A receptor for green tea polyphenol EGCG. Nat Struct Mol Biol 11: 380-381.

26. Mi L, Xiao Z, Hood BL, Dakshanamurthy S, Wang X, et al. (2008) Covalent binding to tubulin by isothiocyanates. A mechanism of cell growth arrest and apoptosis. J Biol Chem 283: 22136-22146.

27. Shibata T, Kimura Y, Mukai A, Mori H, Ito S, et al. (2011) Transthiocarbamoylation of proteins by thiolated isothiocyanates. J Biol Chem 286: 42150-42161.

28. Murakami A, Shigemori T, Ohigashi H (2005) Zingiberaceous and citrus constituents, 1'-acetoxychavicol acetate, zerumbone, auraptene, and nobiletin, suppress lipopolysaccharide-induced cyclooxygenase-2 expression in RAW264.7 murine macrophages through different modes of action. J Nutr 135: 2987S-2992S.

29. Prasannan R, Kalesh KA, Shanmugam MK, Nachiyappan A, Ramachandran L, et al. (2012) Key cell signaling pathways modulated by zerumbone: Role in the prevention and treatment of cancer. Biochem Pharmacol 84: 1268-1276.

30. Sengupta S, Jang BC, Wu MT, Paik JH, Furneaux H, et al. (2003) The RNA-binding protein HuR regulates the expression of cyclooxygenase-2. J Biol Chem 278: 25227-25233.

31. Ohnishi K, Irie K, Murakami A (2009) In vitro covalent binding proteins of zerumbone, a chemopreventive food factor. Biosci Biotechnol Biochem 73: 1905-1907.

32. Shin JW, Ohnishi K, Murakami A, Kundu JK, Na HK, et al. (2011) Zerumbone induces heme oxygenase-1 expression in mouse skin and cultured murine epidermal cells through activation of Nrf2. Cancer Prev Res (Phila). 
33. Guettouche T, Boellmann F, Lane WS, Voellmy R (2005) Analysis of phosphorylation of human heat shock factor 1 in cells experiencing a stress. BMC Biochem 6: 4.

34. Codreanu SG, Zhang B, Sobecki SM, Billheimer DD, Liebler DC (2009) Global analysis of protein damage by the lipid electrophile 4-hydroxy-2-nonenal. Mol Cell Proteomics 8: 670-680.

35. Pérez VI, Buffenstein R, Masamsetti V, Leonard S, Salmon AB, et al. (2009) Protein stability and resistance to oxidative stress are determinants of longevity in the longest-living rodent, the naked mole-rat. Proc Natl Acad Sci U S A 106: 3059-3064. 